JUAN CARLOS ORTIZ NICOLÁS

INSTITUTO DE ARQUITECTURA, DISEÑO Y ARTE

UNIVERSIDAD AUTÓNOMA DE CIUDAD JUÁREZ

CIUDAD JUÁREZ, CHIHUAHUA, MÉXICO

CARLOS.ORTIZ@UACJ.MX
Fecha de recepción: 17/01/2018

Fecha de aceptación: 21/03/2018

Cómo citar: ORTIZ, J. (2018)

Analizando respuestas verbales para identifi-

car evaluaciones temáticas en la interacción

persona-objeto. RChD: creación y pensamiento,

3(4), 1-13.

DOI: $10.5354 / 0719-837 \times .2018 .49816$

\section{Analizando respuestas verbales para identificar evaluaciones temáticas en la interacción persona-objeto}

\author{
Analysing verbal data to identify appraisals themes \\ in the person-object interaction
}

Resumen. Esta investigación presenta cómo analizar respuestas verbales con el fin de identificar evaluaciones temáticas de eventos que activan emociones particulares en la interacción persona-objeto. Las evaluaciones temáticas son descripciones generales que sintetizan y reflejan respuestas similares, a partir de reportes subjetivos de un grupo de personas al experimentar una emoción particular. Dos emociones: confianza y frustración son utilizadas como casos de estudio, con el objetivo de identificar sus evaluaciones temáticas. El artículo presenta una descripción procedimental para obtener la información y detalla cómo realizar el análisis de los datos obtenidos. Identificar las evaluaciones temáticas que activan una emoción ayuda a que especialistas en diseño entiendan mejor la experiencia emocional que las personas tienen con los objetos. Las evaluaciones temáticas también son puntos de partida para diseñar emociones particulares.

Palabras clave: Análisis de resultados, diseño para las emociones, experiencia de usuario, teoría cognitiva de las emociones.

Abstract. This research introduces how to analyze verbal data to identify appraisals themes that trigger specific emotions in the person-object interaction. Appraisal themes are general descriptions that synthesize and reflect similar responses that a group of people experience in particular emotions. Two emotions: confidence and frustration are used as case studies to identify their appraisal themes. The article presents the general process to obtain the data and details on how to undertake the analysis. Identifying appraisal themes helps designers understand the emotional experience that people have with objects. It can also be the starting point to design for eliciting specific emotions.

Keywords: Appraisal theory, data analysis, design for emotions, user experience.

Revista Chilena de Diseño

RChD: creación y pensamiento

Universidad de Chile

2018, 3(4)

http://rchd.uchile.cl 
1. El término appraisal puede traducirse como

evaluación o valoración. En este artículo hemos optado por la primera.

\section{Introducción}

Estudiar las emociones en el diseño es relevante porque estas son una característica propia del ser humano; pensar en ellas implica que especialistas en diseño retomen el rol central que tienen las personas en su interacción con objetos. Investigaciones en el campo de la experiencia de usuario ha identificado que las emociones tienen un rol básico para definir una experiencia como placentera o no placentera (McCarthy y Wright, 2004; Hassenzahl, 2010). De igual manera, son un área de oportunidad para estimular la creatividad de especialistas en diseño (Desmet, 2012; Ortiz Nicolás, Aurisicchio y Desmet, 2013). Otra razón que hace interesante estudiarlas, es que las emociones están asociadas o ancladas a algo (Scherer, 2005; Scherer, Schorr y Hohnstone, 2001), lo que es de gran utilidad para identificar qué las desencadena o activa. En el contexto de esta investigación cómo un objeto (producto, servicio o interfaz) es detonador de emociones.

Si bien, existen diferentes teorías que abordan factores particulares de las emociones (ver Meiselman, 2016), esta investigación se alinea con la teoría cognitiva, que en su forma más simple afirma que las emociones son provocadas por evaluaciones (valoraciones) ${ }^{1}$ de eventos y situaciones (Roseman y Smith, 2001). Por lo que todas las situaciones a las que se asigna el mismo patrón de evaluación evocarán la misma emoción; por ejemplo, la pérdida de un objeto valorado genera tristeza (Lazarus, 2001). Dichos patrones se denominan evaluaciones temáticas. Debe considerarse que un número indeterminado de situaciones que se evalúan de manera similar evocarán la misma emoción, incluidas las situaciones no experimentadas previamente (Roseman y Smith, 2001, p. 7). Podemos afirmar que una gran diferencia de la teoría cognitiva, respecto a otras, es que considera las evaluaciones como punto de partida de la experiencia emocional (Scherer et al., 2001). En el ejemplo previo de la tristeza, la pérdida del objeto valorado es la causa que desencadena la emoción. Cada teoría de las emociones tiene objetivos particulares, por ejemplo, la teoría de las emociones universales (ver Ekman, 1992) trata de identificar gestos, sonidos, posturas y movimientos del cuerpo representativos de emociones concretas y que se replican en diferentes culturas (Ekman, 1992; Jacob-Dazarola, Ortiz Nicolás y Cárdenas Bayona, 2016); sin embargo, no profundiza en aquello que inicia el proceso de experimentar una emoción. En contraparte, la teoría cognitiva sostiene que existen patrones comunes de evaluación que evocan o activan una misma emoción en varias personas; dichos patrones los denominamos evaluaciones temáticas. La teoría cognitiva ha sido adoptada en el campo del diseño emocional con anterioridad. Desmet (2002) establece que es óptima para la práctica del diseño porque ayuda a identificar qué activa o estimula una emoción en la interacción persona-producto, lo que, a su vez, es una oportunidad para el diseño emocional porque ayuda a establecer mayor control en el proceso para evocarlas (Desmet, 2002; Ortiz Nicolás, Aurisicchio y Desmet, 2013a; Yoon, Pohlmeyer y Desmet, 2016). Las evaluaciones temáticas representan fines concretos para el diseño emocional, por ejemplo, crear objetos que permitan a las personas crecer y desarrollar actividades significativas, tiene el potencial de evocar inspiración (Ortiz Nicolás, Aurisicchio y Desmet, 2013a). Así, los intentos para evocar una emoción particular en la interacción persona-producto pueden facilitarse mediante la comprensión del patrón de evaluación que estimula dicha emoción (Demir et al., 2009). Lo anterior es significativo debido a que existe investigación previa que ha establecido 
que al diseñar emociones en el campo profesional se recurre generalmente a técnicas de prueba y error o a la intuición del diseñador (Maya Castaño, 2017; Ortiz Nicolás, Aurisicchio y Desmet, 2013; Porter, Chhibber y Porter, 2008). Los resultados de la investigación en el campo de la teoría cognitiva justifican la viabilidad de identificar qué activa una emoción concreta en la interacción persona-producto. Una limitante de la investigación previa es que los métodos para analizar e identificar las evaluaciones que activan emociones se han mantenido en el campo académico, considerando que la investigación en diseño generalmente recae en investigadores profesionales o en formación (profesores y estudiantes de doctorado). Es importante que el conocimiento generado en la academia permee hacia otros grupos: profesionales o estudiantes de licenciatura porque el tema de las emociones y afectividad es de interés para la industria (Mahut et al., 2018).

El objetivo de esta investigación es presentar un método procedimental para analizar datos obtenidos a través de respuestas verbales, con el fin de identificar y sugerir evaluaciones temáticas que reporten cómo se activan emociones particulares en la interacción persona-objeto. Por lo tanto, se cumplirá el objetivo al presentar evaluaciones temáticas que activan o desencadenan una emoción particular a partir de los datos recabados.

La presente investigación se desarrolla en cinco secciones principales, la primera presenta características fundamentales de las emociones, y explica el concepto de evaluación temática; la segunda presenta el estudio desarrollado, la tercera refiere los resultados, la cuarta despliega la discusión del estudio y, finalmente, la quinta da a conocer las conclusiones.

\section{Marco teórico}

Las emociones son fenómenos complejos y multi-compuestos o multi-estructurados (Fredrickson 2003; Scherer, 2005). Scherer (2005), por ejemplo, ha identificado siete características: son eventos enfocados, impulsadas por una evaluación, con respuesta sincronizada, con rapidez de cambio, impacto conductual, involucran un factor de intensidad (calmado-excitante) y tienen corta duración. Considerando el interés de esta investigación se retoman las dos primeras características establecidas por Scherer: los eventos enfocados son los objetos (producto, servicio, interfaz) y el fin es identificar las evaluaciones temáticas que las estimulan o activan.

En el estudio general de las emociones se ha identificado que estas pueden ser causadas por al menos cuatro procesos: 1) neuronales, 2) sensoriomotores, 3) afectivos, y 4) cognitivos (Izard, 1993). Izard presenta evidencia de los cuatro procesos, por ejemplo, algunos componentes bioquímicos que se presentan en el cerebro pueden estimular depresión; el dolor puede causar enojo; sentir orgullo por cumplir una meta relevante para el ser, a su vez, puede desencadenar otra emoción; finalmente, la evaluación de un evento como agradable o desagradable (proceso cognitivo) puede evocar emociones positivas o negativas. La investigación de Izard argumenta que una emoción puede activarse sin la intervención de los procesos cognitivos, y si bien esto es correcto, también confirma que una emoción puede ser causada o desencadenada por procesos cognitivos. Lo anterior coincide con el conocimiento desarrollado en el campo de la psicología y las emociones (Damasio, 2015; Scherer, 2005; Lazarus, 2001; Smith y Lazarus, 1993).

En la teoría cognitiva, los procesos que desencadenan una emoción se denominan appraisals (traducciones cercanas son: valoración y evaluación). 
2. La definición en el idioma original es an act of assessing something or someone.

3. Cita en el idioma original: Appraisal is an

evaluation of what one's relationship to the environment implies for personal well-being. Each positive emotion is said to be produced by a particular kind of appraised benefit, and each negative emotion by a particular kind of appraised harm.
En este artículo se usa 'evaluación' porque consideramos que comunica mejor la idea de appraisal. El Diccionario Oxford indica que appraisal es "un acto de evaluar algo o alguien". ${ }^{2}$ En el campo de la teoría cognitiva, appraisal es una evaluación de la relación que tiene un individuo con el medio ambiente y sus implicaciones para el bienestar personal. Se dice que cada emoción positiva es producida por un tipo particular de beneficio apreciado, y cada emoción negativa por un tipo particular de daño apreciado (Smith y Lazarus, 1993). ${ }^{3}$ Lo anterior implica que las personas pueden establecer conscientemente si algo es benéfico o dañino.

Para el estudio de las evaluaciones que activan emociones existen dos aproximaciones: temáticas y componenciales. Demir et al. (2009) explican que las evaluaciones temáticas se describen en términos de declaraciones resumidas, las cuales reflejan el significado personal general de una situación que evoca una emoción. Lazarus (2001) explica que son categorías generales que sintetizan evaluaciones similares reportadas por un grupo de personas. Las evaluaciones componenciales, por su parte, describen una emoción en términos de los componentes involucrados a partir de una situación, y responden a preguntas tales como: ¿Se relaciona esta situación con mis motivos? ¿En qué medida se esperaba esta situación? ¿Quién o qué es responsable de esta situación? Este proyecto retoma el enfoque de las evaluaciones temáticas por la manera en la que se obtiene la información, la cual no es tan detallada como en las evaluaciones componenciales (para ver ejemplos de este tipo de aproximaciones en el diseño recomendamos Demir y Colegas, 2009 y Ortiz Nicolás, 2014).

Las evaluaciones tienen un rol básico en la activación de una emoción porque cumplen la importante función mediadora de vincular las respuestas emocionales a las circunstancias ambientales, por un lado, y los objetivos y creencias personales, por el otro (Smith y Lazarus, 1993). Finalmente, la teoría cognitiva sugiere que existe más de una evaluación temática que desencadenan una misma emoción en un grupo de personas.

\section{Estudio}

Los datos a analizar fueron recabados a partir de un método de investigación cualitativo que sugerimos y reportamos anteriormente (Ortiz Nicolás, 2018; Lozada et al., 2017). Dicho método ayuda a identificar las emociones, qué objetos particulares estimulan en un grupo de personas. Una síntesis de los pasos básicos del método es la siguiente:

1) Seleccionar un objeto a evaluar (producto, servicio, interfaz).

2) Determinar las características de la población que evaluará el objeto.

3) Entregar 30 tarjetas que presentan nombres de emociones: 14 positivas, 14 negativas y 2 neutras. Las emociones son: admiración, alegría, inspiración, diversión, orgullo, cariño, relajación, confianza, satisfacción, deseo, sorpresa, entusiasmo, fascinación, ilusión (positivas); añoranza, nostalgia (neutras); aburrimiento, frustración, angustia, insatisfacción, asco, miedo, decepción, sorpresa negativa, desagrado, vergüenza, desconfianza, desencanto, enojo, fastidio (negativas).

4) Los participantes seleccionan las emociones que el objeto evaluado les hace sentir.

5) Los participantes explican verbalmente por qué experimentan cada una de las emocionadas seleccionadas. 
Con la técnica anteriormente descrita hemos estudiado el factor emocional en experiencias agradables, desagradables, significativas y de autonomía. La información recabada de las emociones se usó para crear un banco de respuestas, el cual es la fuente de información que usaremos para cumplir el objetivo de este artículo: analizar la información para identificar y proponer evaluaciones temáticas que activan emociones particulares en la interacción persona-objeto. Sin embargo antes de proseguir hacia nuestro objetivo, realizaremos un paréntesis para presentar algunas consideraciones básicas en proyectos de investigación cualitativa: selección de participantes y estímulo a evaluar. Dichos factores -que presentamos a continuación- afectan directamente la calidad de los datos recabados.

\section{Participantes}

La muestra de participantes debe ser homogénea y compartir similitudes críticas relacionadas con la pregunta u objetivo de investigación (DiCicco-Bloom y Crabtree, 2006). Para establecer el número de participantes recurrimos a la teoría de saturación, que es el punto en la recopilación y análisis de datos en el cual la información nueva produce poco o ningún cambio en el libro de códigos (Guest, Bunce, y Johnson, 2006). En nuestra experiencia el punto de saturación en el reporte de emociones persona-objeto ocurre entre los $15 \mathrm{y}$ 20 participantes, evaluando un solo producto. Cada objeto evaluado requiere un grupo de participantes particular.

\section{Estímulo a evaluar}

Este factor es básico, el especialista en diseño establece el objeto de acuerdo a sus intereses o los de la empresa; por ejemplo, evaluar el prototipo del proyecto

que se está desarrollando o el producto más reciente que salió al mercado.

\section{Recopilación de estudios para este estudio}

Para realizar el análisis de resultados que presentamos en la siguiente sec-

ción hemos generado una base de datos de respuestas. Fueron 75 personas, de entre 20 y 40 años de edad y una población balanceada de hombres y mujeres los que participaron en los estudios que alimentan la base de datos. Todos de nacionalidad mexicana residentes en dos ciudades: de México y Juárez. La lengua madre de todos los participantes fue el español. Los datos que recabamos están asociados a productos durables, aquellos que tienen una vida útil de al menos seis meses, como tijeras, computadoras, teléfonos celulares, automóviles, consolas de videojuegos, etc. Los participantes tenían un conocimiento detallado de los productos seleccionados y evaluados, ya que en la mayoría de los casos eran sus dueños.

Para cumplir el objetivo de este estudio, nos enfocamos en las explicaciones de dos emociones concretas para ejemplificar cómo se realiza el análisis de las respuestas verbales. Las emociones seleccionadas fueron confianza y frustración, considerando los siguientes criterios:

- Las dos emociones son experimentadas con los productos y consideran la valencia, una es positiva y otra negativa (ver Ortiz Nicolás y Hernández López, 2008; Desmet, 2002).

- Existen antecedentes teóricos en relación con las evaluaciones temáticas que las desencadenan. 


\section{Resultados}

En esta sección presentaremos 10 respuestas obtenidas de una base de datos en donde un grupo de personas reportó verbalmente el porqué un objeto les hizo experimentar confianza o frustración. La selección de las 10 respuestas fue al azar. Es importante aclarar que en proyectos en los que se evalúa solo un producto (p.e. una cámara fotográfica) se analizan todas las respuestas de las emociones frecuentemente experimentadas, en donde frecuente significa que fueron reportadas por al menos el $50 \%$ de los participantes.

\section{Confianza}

La Tabla I presenta los resultados para la emoción confianza. Como se dijo, se presentan respuestas a partir de diferentes objetos: tijeras, electrónicos, bolígrafos, etc. Esto es válido para este estudio porque el objetivo es analizar e identificar evaluaciones temáticas que activan la emoción confianza.

Tabla 1. Evaluaciones que estimulan confianza

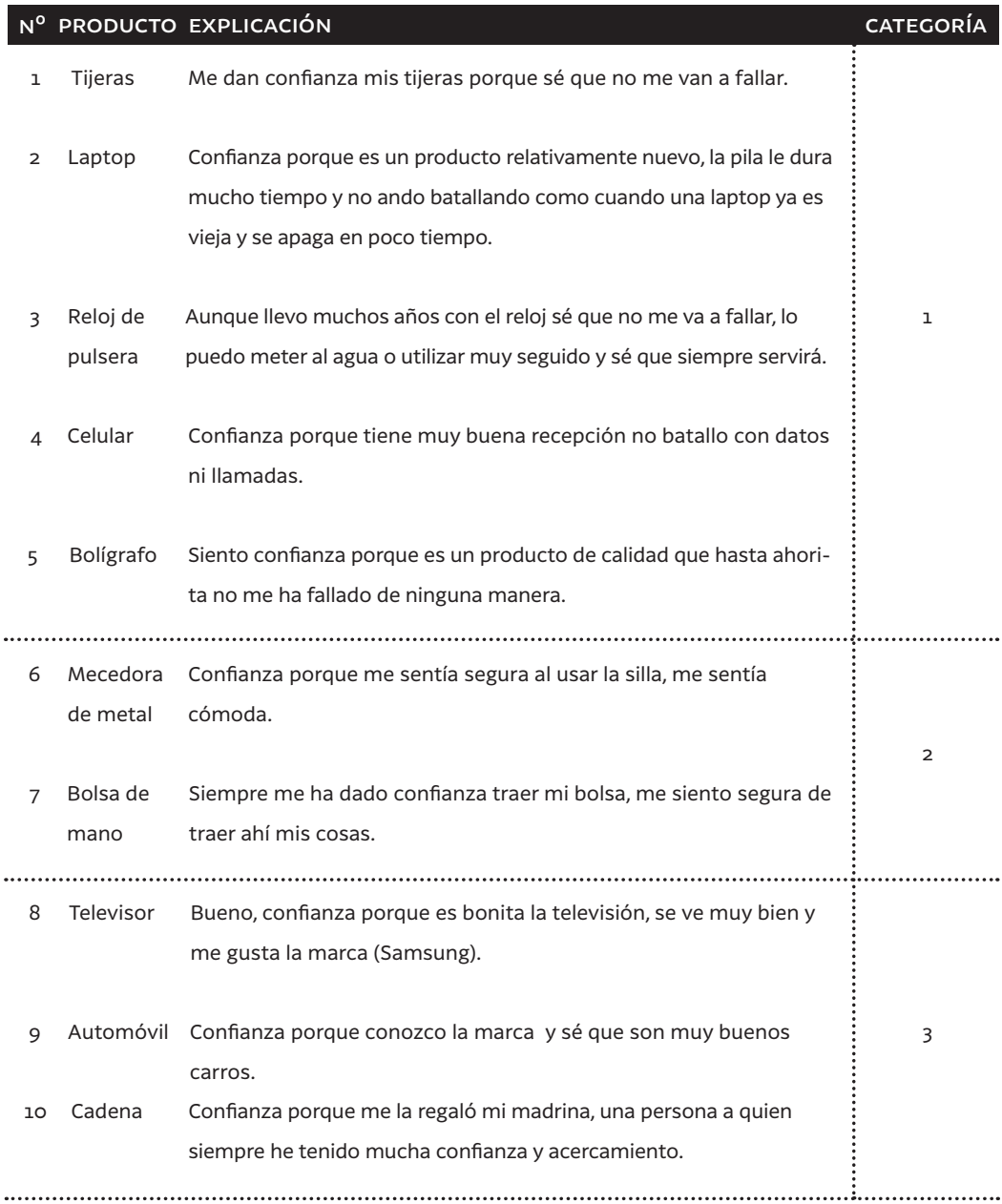


Considerando que este estudio es cualitativo, se recurrió a los pasos básicos que sugiere Fernández Núñez (2006) para realizar el análisis de resultados. El objetivo del análisis es claro: identificar causas similares que activan confianza o frustración. Fernández Núñez sugiere:

I. Obtener la información. Un grupo de personas explicó verbalmente por qué experimentaron una emoción particular, en este caso concreto retomamos 10 respuestas.

II. Capturar, transcribir y ordenar la información. En esta investigación nos centramos en el factor emocional, ya que los estudios profundizaban en otras dimensiones de la experiencia.

III. Codificar la información. Codificar es el proceso mediante el cual se agrupa la información obtenida en categorías que concentran las ideas, conceptos o temas similares descubiertos por el investigador, o los pasos o fases dentro de un proceso (Rubin y Rubin en Fernández Núñez, 2006). Las agrupaciones pueden identificarse en las Tablas 1 y 2 en la columna categoría. Las categorías se establecieron a partir de explicaciones similares al experimentar una emoción. Por ejemplo, la Tabla 1 integra cuatro respuestas para la categoría 1, que están asociadas al buen desempeño de la función instrumental del producto. Cada categoría se reporta en una evaluación temática.

IV. Integrar la información. Relacionar las categorías obtenidas en el paso anterior, entre sí y con los fundamentos teóricos de la investigación (Fernández Núñez, 2006).

Las fases más complejas para el análisis de resultados son la III y IV. Es importante tener presente que estas fases se influencian recíprocamente, al categorizar se toman decisiones que después se reflejarán en la integración. Es un proceso en el cual el investigador identifica las categorías y las integra, considerando interrogantes como: ¿La categoría y su integración son las adecuadas? ¿La integración comunica claramente las similitudes y diferencias que reportaron las personas? ¿Cuántas categorías se requieren para reportar adecuadamente los resultados obtenidos? A partir de los datos presentados en la Tabla 1 se identificaron tres evaluaciones temáticas para confianza:

1) Las personas experimentan confianza cuando el producto tiene un desempeño excelente en su función instrumental; ${ }^{4}$ esto les hace creer que nunca va a fallar. El producto cumple su función sin contratiempos, p.e., las tijeras cortan bien.

2) Las personas experimentan confianza cuando se sienten seguras y cómodas al usar el producto, p.e., sentí comodidad y seguridad al usar la silla.

3) Las personas experimentan confianza cuando las marcas tienen buena reputación en relación a los productos que ofrecen, son de calidad y sirven. Una participante menciona que el producto le recuerda a la persona que se lo regaló y en quien la participante confía. Un proceso similar ocurre con las marcas.
4. Se ha sugerido que los productos cumplen varias funciones, instrumentales, estéticas y simbólicas, en este caso nos referimos a las instrumentales, un vaso contiene agua (ver Crilly, 2010; Aurisicchio et al., 2011). 
Al realizar el análisis identificamos que había diferencias sutiles entre la primera y segunda evaluación temática. En la primera se hace un fuerte énfasis en la función instrumental y en la segunda en la seguridad y comodidad. Anteriormente mencionábamos que el proceso de categorización e integración se influencian recíprocamente, y este es un buen ejemplo para clarificar nuestro punto: sintetizar los datos injustificadamente afecta directamente su riqueza.

\section{Frustración}

La Tabla 2 presenta 10 razones que un grupo de personas reportó al experimentar frustración.

Tabla 2. Evaluaciones que estimulan frustración

\section{$\mathrm{N}^{\circ}$ PRODUCTO CAUSA}

Lámpara
Insatisfacción porque ya no me gusta el color y por lo mismo que no puedo dosificar la luz, fastidio, porque de repente tanta luz ya no me es placentera tan noche y frustración de que no puedo cumplir como con esa necesidad [ajustar la intensidad].

\footnotetext{
2 Celular
} Frustración cuando se pone lento y me saca de mis aplicaciones.

3 Reproduc- Frustración porque se atora cada 30 canciones. tor $\mathrm{Mp}_{3}$

4 Cámara fotográfica

Frustración porque a la hora de tomar la foto y enfocar, pues no la toma bien o se traba demasiado.
5 Disco compacto

6 Mochila

(lateral)

7 Consola de videojuegos

8 Mochila

Frustración porque cuando se raya no puedes escuchar tu música y es lo que necesitas para relajarte.

Es frustrante no tener con qué llevar bien tus cosas, estar con todas las cosas amontonadas y no tener con qué llevarlas correctamente.

Frustración, yo digo que también se explica por sí solo, porque, si no sale algo bien es igual que el enojo que no puedes pasar una misión o que se apague, o cualquier fallita.

Bueno, primero fastidio porque es incómodo cargarla, no puedo cargarla a gusto como normalmente debería de ser y luego frustración viene de lo mismo del fastidio porque ya de tanto que la uso a diario y camino se podría decir, un largo recorrido, entonces me frustra no poder cargar cómodamente la mochila.

9 Zapatos Frustración porque al momento de tener puestos los tenis no estas cómodo.

10 Tenis Como no me gusta físicamente y es algo que tengo que vestir me frustra. 
A partir de los datos presentados en la Tabla 2 se identificaron cuatro evaluaciones temáticas para frustración:

1) Cuando el producto no les permite desempeñar una tarea deseada, p.e. ajustar la intensidad de la luz.

2) Cuando el producto desempeña la tarea de manera deficiente, p.e. capturar una fotografía borrosa.

3) Cuando al usarlo un objeto les genera incomodidad, p.e. usar zapatillas apretadas.

4) Las personas experimentan frustración cuando son obligadas a usar un objeto que no les gusta, en este caso una prenda de vestir.

Al realizar el análisis cualitativo, en particular la categorización e integración, identificamos que era importante reportar que una persona experimenta frustración al no poder realizar una tarea deseada y también cuando un producto permite realizar la tarea, pero el resultado es inadecuado. Existen diferencias sutiles en ambos casos que son relevantes de reportar.

\section{Discusión}

Este artículo presentó un método procedimental para analizar respuestas verbales con el fin de identificar y sugerir evaluaciones temáticas que reportan cómo se activan emociones particulares en la interacción persona-objeto. El fin es que el proceso de evaluación y análisis de emociones sea implementado no solo en el ámbito académico, sino también en el profesional. Los resultados de esta investigación confirman que, a partir de descripciones verbales que un grupo de personas reportó con relación a emociones concretas, es posible identificar las evaluaciones temáticas que las activan.

Si bien las respuestas que se presentan en el estudio emergen de la interacción con una gama amplia de productos, se identificaron evaluaciones similares que se sintetizaron en evaluaciones temáticas. Es significativo reportar que a partir de diez respuestas, fue posible identificar coincidencias en las evaluaciones que activan confianza o frustración. Hay que recordar, sin embargo, que la teoría cognitiva propone que existe un número indeterminado de evaluaciones que desencadenan una emoción, en este caso identificamos tres para confianza y cuatro para frustración. Lo anterior no significa que argumentemos que se han identificado todas las evaluaciones temáticas. Es probable que nuevos estudios en el área de la interacción persona-producto enfocados en la confianza o frustración, puedan corroborar nuestros hallazgos y complementarlos.

Una vez presentados los resultados podemos contrastarlos con evaluaciones temáticas que han sido sugeridas en investigaciones previas acerca de estos tópicos. Desmet (2012, p. 4) sugiere dos evaluaciones temáticas para experimentar confianza; la primera al sentir fe en uno mismo o en sus habilidades para lograr o actuar correctamente; la segunda, cuando los productos son claros y fáciles de usar (Desmet, 2012, p. 10). Otras investigaciones identificaron que productos con excelente función instrumental generan confianza (Ortiz Nicolás, Aurisicchio y Desmet, 2013). De tal manera, los resultados obtenidos en México tienen una coincidencia parcial con la de otros países. Por un lado, no se identificaron evaluaciones temáticas ligadas a la fe en uno mismo y sus habilidades para actuar correctamente. La explicación que damos es que nuestra muestra de respuestas es mucho menor que la utilizada por Desmet, y esto se debe a que cada investigación tiene objetivos particulares. 
Por otro lado, sí identificamos que el excelente desempeño de la función instrumental de un producto es un factor fundamental para experimentar confianza, como se ha establecido en Holanda e Inglaterra y ahora México. Lazarus (2001) menciona que las personas experimentan frustración al ver que un evento deseado todavía no se ha materializado. Esto tiene una clara relación con la primera evaluación temática identificada en esta investigación; si las personas no pueden realizar lo que quieren experimentan frustración. La línea, sin embargo, es más difusa con los productos porque no se reduce al no poder hacer. Mencionamos lo anterior porque también identificamos que si un producto funciona de forma deficiente al completar una tarea deseada o genera incomodidad en su uso, causa frustración. Punto y aparte, es el hecho de obligar a una persona a hacer algo que no quiere. Identificar y conocer las evaluaciones temáticas son áreas de oportunidad para diseñar (ver Ortiz Nicolás et al., 2013a; Desmet, 2012; Yoon et al., 2016), por lo que identificar evaluaciones temáticas se une a otras estrategias que consideran a las emociones en la práctica del diseño (Jacob-Dazarola, 2017; Maya-Castaño, 2017). Las evaluaciones temáticas, además, tienen el potencial de complementar métodos que consideran las emociones en la práctica del diseño (ver Ortiz Nicolás et al., 2013a; Desmet, 2002). Argumentamos lo anterior basándonos en los hallazgos de Daalhuizen (2014). Él identificó que existen tres grandes factores en la enseñanza de métodos de diseño: el diseñador con su conocimiento, la tarea de diseño y el contexto en donde se desarrolla dicha tarea. El contexto incluye herramientas y conocimiento de apoyo que recibe el diseñador/a. Estar familiarizado con las evaluaciones temáticas impacta el conocimiento del especialista en diseño, ya que es más preciso y evita que él o ella se coloque como referencia única al diseñar. Conocer las evaluaciones temáticas, además, tiene el potencial de desarrollar la granularidad emocional en especialistas en diseño; es decir, reconocer las pequeñas diferencias entre la amplia gama de emociones que experimentamos (Yoon et al., 2016). Lo anterior justifica que tanto académicos como profesionales desarrollen conocimiento de las emociones relevantes a la práctica del diseño. Este artículo trata de promover que se alcance dicha meta. Un factor básico para diseñar emociones es establecer el medio para lograrlo, que en esta investigación es el objeto (producto, servicio, interfaz). De tal manera, el diseñador debe reflexionar si es importante que las personas experimenten emociones particulares, como la confianza o frustración, en el objeto a desarrollar. El equipo de diseño debe establecer qué emociones son relevantes para el proyecto a desarrollar. Maya Castaño (2017) propone al menos tres consideraciones para lograrlo: i) lo esperado y deseado por el usuario, ii) los intereses del especialista en diseño, y iii) la estrategia que busca el producto y marca. Estas pautas pueden ayudar a definir la experiencia emocional adecuada para el objeto a desarrollar.

Los resultados de este estudio indican que una manera de evitar la frustración en el usuario es que este logre completar la tarea que quiere. Sin embargo, a menudo los diseñadores fallamos en cumplir ese objetivo; tan solo hay que revisar la gran cantidad de quejas que existen contra algunos productos, como las páginas web de varias naciones. Además, evitar que una persona experimente emociones negativas no implica que automáticamente experimente emociones positivas, de la misma manera que evitar una experiencia desagradable no genera una agradable (Hassenzahl, 2010). Evitar que las personas experimenten frustración no genera automáticamente 
alegría, diversión, sorpresa o confianza, tal y como se puede corroborar con las evaluaciones temáticas identificadas en este estudio.

En este artículo nos hemos enfocado en el análisis de los resultados, a partir de respuestas verbales de la experiencia emocional en la interacción persona-objeto. Consideramos que el proceso de análisis es de utilidad para que especialistas en diseño desarrollen habilidades básicas en investigación cualitativa. Promover esta habilidad, además, podría impactar la práctica profesional en al menos dos vías. La primera, identificar evaluaciones temáticas es una opción a la introspección, una estrategia a la que se recurre para diseñar emociones y que es poco confiable (ver Maya-Castaño, 2017; Ortiz Nicolas et al., 2013; Porter et al., 2008). La segunda, al estimular la práctica reflexiva, actividad recurrente en la disciplina del diseño (Schön, 1992). Entender la realidad de otras personas es básico para reflexionar sobre la experiencia personal, ¿las evaluaciones temáticas son similares en mi ser?

La práctica reflexiva también se estimula en el diseño emocional.

El estudio de las emociones ayuda a entender la experiencia del usuario, la cual se modifica a través del tiempo (Kujala et al., 2011; Karapanos et al., 2009). Una persona que interactúa con un mismo objeto por un período determinado puede experimentar varias emociones (diferentes o similares): al tener contacto inicial con el objeto puede experimentar desconfianza porque desconoce su funcionamiento. Después de un proceso de familiarización la persona podría experimentar satisfacción. Finalmente, cuando el objeto deja de funcionar y tiene que desecharlo, la persona podría experimentar tristeza. La teoría cognitiva de las emociones puede ayudar a entender cómo se modifica la experiencia del usuario a través del tiempo.

Una limitación de esta investigación es que se enfoca en el nivel cognitivo, al recurrir a explicaciones verbales de las causas que activan o desencadenan una emoción. Las emociones son fenómenos complejos que se experimentan de forma integral (Scherer, 2005; Damasio, 2015), es decir, experimentarlas no ocurre solo en el nivel cognitivo, sino que existen implicaciones a nivel químico, fisiológico y conductual (Izard, 1993). Damasio (2015, p. 224) menciona que los sentimientos nos permiten prestarle atención al cuerpo, de manera concentrada, como en un estado emocional, o vagamente, como durante un estado de fondo. Encontramos resonancia en Damasio, porque a pesar de enfocarnos en respuestas verbales, factores somáticos emergieron en los temas de evaluación identificados. Por ejemplo la incomodidad (factor físico) puede generar frustración y la comodidad confianza. Es fundamental que especialistas en diseño consideren a las personas de forma integral, incluyendo su corporeidad, tanto en el estudio de usuarios, como en la práctica del diseño.

\section{Conclusiones}

Los resultados de este estudio corroboran que es posible identificar evaluaciones temáticas que activan o desencadenan una emoción en la interacción persona-producto, a partir de explicaciones verbales. Dichas evaluaciones pueden convertirse en objetivos o metas concretas a lograr por especialistas durante el desarrollo proyectual. Lo anterior justifica que, tanto académicos como profesionales, desarrollen habilidades para identificar evaluaciones temáticas de emociones relevantes a los proyectos que están desarrollando. Los resultados presentados en este artículo pueden ayudar a especialistas en diseño a identificar nuevas oportunidades para el desarrollo de objetos al considerar las emociones. También, a ser más rigurosos en la enseñanza y 
práctica del diseño emocional superando factores que, si bien son relevantes, como la introspección, no siempre son confiables. Conocer cómo otras personas experimentan una emoción es de utilidad para que especialistas en diseño reflexionemos respecto a nuestras experiencias individuales y las comparemos a partir de conocimiento desarrollado por la investigación. Las emociones son un factor central en la experiencia del usuario, lo que justifica estudiarlas con profundidad, teniendo presente que la afectan directamente y que la experiencia, a su vez, está interconectada con otros factores también importantes, como la consciencia, la subjetividad, la temporalidad y la corporeidad.

\section{Referencias}

Aurisicchio, M., Eng, N. L., Ortiz Nicolás, J. C., Childs, P., \& Bracewell, R. H. (2011). On the functions of products. In DS 68-10: Proceedings of the 18th International Conference on Engineering Design (ICED 11), Impacting Society through Engineering Design, vol. 10: Design Methods and Tools pt. 2, Lyngby/Copenhagen, Denmark, 15. [19.08.2011].

Crilly, N. (2010). The roles that artefacts play: technical, social and aesthetic functions. Design Studies. doi:10.1016/j.destud.2010.04.002

Daalhuizen, J. J. (2014). Method Usage in Design: How methods function as mental tools for designers. Ph.D. Dissertation. Delft University of Technology. The Netherlands.

Damasio, A. R. (2015). El error de Descartes. La emoción, la razón y el cerebro humano. Ciudad de México: Booket.

Demir, E., Desmet, P. M., \& Hekkert, P. (2009). Appraisal patterns of emotions in human-product interaction. International Journal of Design, 3(2).

Desmet, P. M. (2012). Faces of product pleasure: 25 positive emotions in human-product interactions. International Journal of Design, 6(2), 2012.

Desmet, P. M. A. (2002). Designing Emotions. Unpublished doctoral dissertation. Delft University Of Technology, The Netherlands.

DiCicco-Bloom, B., \& Crabtree, B. F. (2006). The qualitative research interview. Medical education, 4O(4), 314-321.

Ekman, P. (1992). An argument for basic emotions. Cognition ef emotion, 6(3-4), 169-200.

Fernández Núñez, L. (2006). Fichas para investigadores. ¿Cómo analizar datos cualitativos? Bulletí LaRecerca, 1-13. http://doi. org/ISSN: 1886-1946 / Depósito legal: B.20973-2006

Guest, G., Bunce, A., \& Johnson, L. (2006). How Many Interviews Are Enough? Field Methods, 18(1), 59-82. http://doi.org/10.1177/1525822X05279903

Hassenzahl, M. (2010). Experience Design: Technology for All the Right Reasons. San Rafael, CA: Morgan \& Claypool Publishers. [eBooks]
Izard, C. E. (1993). Four systems for emotion activation: Cognitive and noncognitive processes. Psychological review, 100(1), 68 .

Jacob-Dazarola, R. (2017). Aquello que esperamos de las cosas: la afectividad como recurso para el diseño de productos. En Ortiz Nicolás, J. C. (Ed.). Afectividady Diseño (pp. 128-167). Ciudad de México: UNAM.

Jacob-Dazarola, R., Ortiz-Nicolás, J. C., \& Cardenas, L. (2016). Behavioral Measures of Emotion. In Meiselman H. (Ed.). Emotion Measurement (pp. 101-124). Amsterdam: Woodhead Publishing.

Karapanos, E., Zimmerman, J., Forlizzi, J., \& Martens, J. B. (2009). User experience over time: an initial framework. In Proceedings of the SICCHI conference on human factors in computing systems (pp. 729-738). ACM. April.

Kujala, S., Roto, V., Väänänen-Vainio-Mattila, K., Karapanos, E., \& Sinnelä, A. (2011). UX Curve: A method for evaluating long-term user experience. Interacting with Computers, 23(5), 473-483.

Lazarus, R. S. (2001). Relational meaning and discrete emotions. Appraisal processes in emotion: Theory, methods, research (pp. 37-67). Oxford, MA: Oxford University Press.

Lozada-Cantorán, C.; Ortiz-Nicolás, J. C., Cortés-López, E. M. (2017). Control percibido, emociones y experiencias satisfactorias. Economía Creativa, o8 [noviembre-abril] (pp. 11-53). Recuperado de: http://centro.edu.mx/ojs_ol/ index.php/economiacreativa/article/view/150/107

Mahut, T., Bouchard, C., Omhover, J. F., Favart, C., \& Esquivel, D. (2018). Interdependency between user experience and interaction: a kansei design approach. International Journal on Interactive Design and Manufacturing (IIIDeM), 12(1), 105-132.

Maya-Castaño, J. H. (2017). D+E: fundamentos, problemática y soluciones pedagógicas. Economía Creativa, 08 [noviembre - abril] (pp. 115-157). [online] Recuperado de: http://centro.edu.mx/ojs_ol/index.php/economiacreativa/article/view/153/110 
McCarthy, J., \& Wright, P. (2004). Technology as Experience, London: The MIT Press.

Meiselman, H. L. (Ed.). (2016). Emotion Measurement. Cambridge, MA: Woodhead Publishing.

Ortiz Nicolás, J. C. (2014). Understanding and designing pleasant experiences with products. Doctoral dissertation, Imperial College London, London, UK.

Ortiz Nicolás, J. C., \& Hernández López, I. (2018). Emociones específicas en la interacción persona-producto: un método de identificación causal. Economía Creati$v a$, abril-noviembre.

Ortiz Nicolás, J.C., Aurisicchio, M., \& Desmet, P. (2013). Differentiating positive emotions elicited by products; An exploration of perceived differences between 25 positive emotions by users and designers. In DS 75-7: Proceedings of the 19th International Conference on Engineering Design (ICED13), Design for Harmonies, vol. 7: Human Behaviour in Design. Seoul, Korea.

Ortiz Nicolás, J. C., Aurisicchio, M., \& Desmet, P. (2013a). Designing for anticipation, confidence, and inspiration. In Proceedings of the 6th International Conference on Designing Pleasurable Products and Interfaces (pp. 31-40). ACM.

Ortiz Nicolás, J. C., \& Hernández López, I. (2008). “Product Relevant Emotions in the Spanish Language". Proceedings of the 6th International Conference on Design of Emotion, Hong Kong, China.
Porter C. S., Chhibber S., \& Porter, J. M. (2008). What makes you tick - an investigation of the pleasure needs of different population segments. In Desmet, P. M. A., Erp V. J., \& Karlsson, M. (Eds.), Design and Emotion Moves (pp. 324-361). Cambridge: Cambridge Scholars Publishing.

Roseman, I. J., \& Smith, C. A. (2001). Appraisal theory. Appraisal processes in emotion: Theory, methods, research (pp. 3-19). Oxford, UK: Oxford University Press. Scherer, K. R. (2005). What are emotions? And how can they be measured? Social science information, 44(4), 695-729.

Scherer, K. R., Schorr, A., \& Johnstone, T. (Eds.) (2001). Appraisal processes in emotion: Theory, methods, research. Oxford, UK: Oxford University Press.

Schön, D. A. (1992). La formación de profesionales reflexivos: Hacia un nuevo diseño de la enseñanza y el aprendizaje en las profesiones. España: Ministerio de Educación y Ciencia.

Smith, C. A., \& Lazarus, R. S. (1993). Appraisal components, core relational themes, and the emotions. Cognition of Emotion, 7(3-4), 233-269.

Yoon, J., Pohlmeyer, A. E., \& Desmet, P. M. A. (2016). When 'feeling good' is not good enough: Seven key opportunities for emotional granularity in product development. International Journal of Design, 10(3), 1-15. 